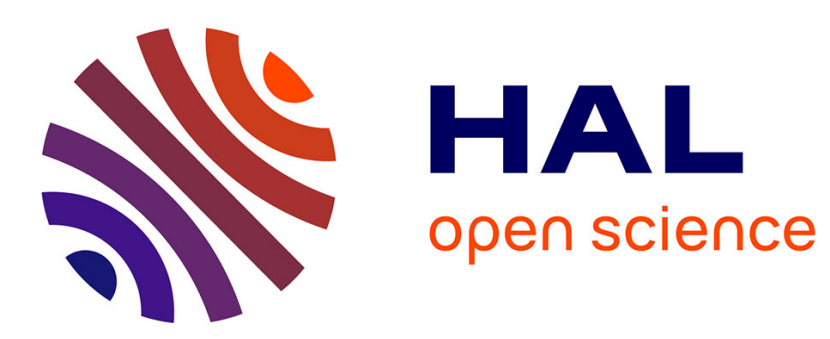

\title{
Block design reconstruction skills: not a good candidate for an endophenotypic marker in autism research
}

\author{
Maretha Jonge, Chantal Kemner, Fabienne Naber, Herman Engeland
}

\section{To cite this version:}

Maretha Jonge, Chantal Kemner, Fabienne Naber, Herman Engeland. Block design reconstruction skills: not a good candidate for an endophenotypic marker in autism research. European Child and Adolescent Psychiatry, 2009, 18 (4), pp.197-205. 10.1007/s00787-008-0708-6 . hal-00478068

\section{HAL Id: hal-00478068 \\ https://hal.science/hal-00478068}

Submitted on 30 Apr 2010

HAL is a multi-disciplinary open access archive for the deposit and dissemination of scientific research documents, whether they are published or not. The documents may come from teaching and research institutions in France or abroad, or from public or private research centers.
L'archive ouverte pluridisciplinaire HAL, est destinée au dépôt et à la diffusion de documents scientifiques de niveau recherche, publiés ou non, émanant des établissements d'enseignement et de recherche français ou étrangers, des laboratoires publics ou privés. 
Maretha de Jonge

Chantal Kemner

Fabienne Naber

Herman van Engeland

\section{Block design reconstruction skills: not a good candidate for an endophenotypic marker in autism research}

Received 19 September 2007

Accepted 27 June 2008

Published online: 16 February 2009
M. de Jonge $(\square) \cdot$ C. Kemner

$\mathrm{H}$. van Engeland

Dept. of Child and Adolescent

Psychiatry, UMC Utrecht, HP B01. 201

PO Box 85500, 3508 GA Utrecht

The Netherlands

E-Mail: m.v.jonge@umcutrecht.nl

C. Kemner

Dept. of Neurocognition

Faculty of Psychology

Maastricht University

Maastricht, The Netherlands

F. Naber

Center for Child and Family Studies

Leiden University

Leiden, The Netherlands
Abstract Superior performance on block design tasks is reported in autistic individuals, although it is not consistently found in highfunctioning individuals or individuals with Asperger Syndrome. It is assumed to reflect weak central coherence: an underlying cognitive deficit, which might also be part of the genetic makeup of the disorder. We assessed block design reconstruction skills in high-functioning individuals with autism spectrum disorders (ASD) from multi-incidence families and in their parents. Performance was compared to relevant matched control groups. We used a task that was assumed to be highly sensitive to subtle performance differences. We did not find individuals with ASD to be significantly faster on this task than the matched control group, not even when the difference between reconstruction time of segmented and pre-segmented designs was compared. However, we found individuals with ASD to make fewer errors during the process of reconstruction which might indicate some dexterity in mental segmentation. However, parents of individuals with ASD did not perform better on the task than control parents. Therefore, based on our data, we conclude that mental segmentation ability as measured with a block design reconstruction task is not a neurocognitive marker or endophenotype useful in genetic studies.

Key words high-functioning autism - autism neuropsychology psychology - cognitive style parents

\section{Introduction}

Autism spectrum disorders (ASD) are developmental disorders that are defined on the basis of behavioral symptoms: qualitative impairments in social and communicative development, with restricted and repetitive activities and interests [4]. A large body of evidence suggests that the heritability for autism is high, but finding the genes appeared to be difficult and so far no single gene has been identified [43]. The genetic liability in autism is reflected in behavioral features sometimes found in first degree relatives that are similar but milder to those found in autism. This is referred to as the broader phenotype of autism [6, 8]. The search for genes is hindered by the fact that there is no known neurobiological marker for autism. Numerous studies, therefore, search for neurocogni- 
tive features of the disorder, both in autistic individuals as well as in their first-degree relatives. Neurocognitive characteristics may be more closely linked to underlying brain anomalies and genetic factors than the behavioral phenotype [22].

One of the leading theories of neurocognitive functioning in autism suggests that autism is characterized by a weak central coherence. Weak central coherence refers to a detailed-focussed information processing style in which features are perceived at the expense of the global configuration $[15,16]$. As a consequence, weak central coherence predicts relatively poor performance on tasks requiring the integration of stimuli in context, but good performance on tasks that require attention to details (see [21] for a review). An example of a task that requires the suppression of the global configuration in order to process the information in a detailed fashion is the block design reconstruction subtest of the Wechsler Intelligence Scales. The construction of a block design requires mentally breaking up a pattern in separate parts and then reconstructing it by synthesizing the blocks into the original design. Autistic children and adults were found to perform relatively good on this task $[9,14,17,18,20,37,41,42,44]$. Their superior performance is explained by a specific asset in mentally segmenting the designs. This was shown in an elegant experiment of Shah and Frith [44], in which the reconstruction time of unsegmented and pre-segmented designs were compared within individuals. The reconstruction time difference between these pattern types was significantly smaller in individuals with autism compared to control individuals, denoting better mental segmentation skills. However, in Asperger Syndrome or high-functioning autism relative good performance on block design tasks in comparison to other tasks is not consistently found [12, 23, 32, 35, 41, 49]. Hence, the peak in performance on block design might be related to the cognitive level of individuals with autism.

There is some evidence that weak central coherence is part of the broader phenotype of autism. For example, parents of autistic individuals are found to perform better than control parents on the Embedded Figures Test, a task that requires the perception of details [7, 11, 19]. With regard to block design, superior performance on the block design subtest of the Wechsler scales relative to other subtests was not found in parents of autistic individuals [13, 40, 47]. Yet, Happé and coworkers [19] found mental segmentation superiority using the task of Shah and Frith [44] in fathers of autistic individuals compared to fathers of control families. However, in mothers or siblings of the autistic children, mental segmentation skills were not found to be significantly better than in matched control individuals [19].

Accordingly, although superior performance on block design tasks is frequently found in autistic individuals, it is not consistently found in higher functioning individuals and first degree relatives. The findings in samples including individuals with lower intellectual abilities might have been influenced by other factors such as motivational factors, uneven IQ profiles and difficulties to find a comparison group that is matched on both chronological age and IQ. In the present study, we aimed to investigate whether superior block design reconstruction skills and mental segmentation abilities are indeed phenotypic features of autism and the broader phenotype. We therefore assessed a sample of high-functioning individuals with ASD and their parents and compared them to normal control groups that were matched on sex, age and IQ. In order to maximize the chance for finding a neurocognitive reflection of a genetic liability for ASD, the individuals with ASD and their parents in the present study were all from multi-incidence families, i.e. families in which two or more siblings are diagnosed with ASD.

If weak central coherence is exhibited in superior mental segmentation skills, sensitive tasks would be needed to manifest an endowment in high-functioning individuals. We chose to use a block design task developed by Akshoomoff and Stiles [3], which has the advantage that it consists of different pattern types that vary in level of complexity and perceptual cohesiveness. We presented them in an unsegmented and a pre-segmented condition, according to the task used by Shah and Frith [44] in order to tease out mental segmentation ability. We videotaped all assessments in order to make blind rating possible and to enable the use of a computer program for accurate measurement of the reconstruction time and errors made.

We hypothesized that, compared to control individuals, high-functioning individuals with ASD and their parents, would show superior performance in reconstructing the designs especially in the most difficult condition with the highest level of perceptual cohesiveness, requiring the greatest amount of mental segmentation ability. We also expecteded the differences in performance between unsegmented and segmented patterns to be smaller in individuals with ASD and their parents than in the matched control groups. Since previous studies found behavioral and cognitive characteristics of the broader phenotype to be more prominent in males than in female relatives $[5,6,8,19,38,39,48]$ we expected to find enhanced performance mainly in 
the group of fathers and probably not in the group of mothers.

\section{Method}

\section{Participants}

Families with at least two children with ASD who participated previously in a genetic study of autism (by the International Molecular Genetic Study of Autism Consortium) were invited to take part in the present study. Recruitment for this previous genetic study took place in the outpatient and inpatient clinics of the Department of Child and Adolescent Psychiatry of the Academic Medical Centre in Utrecht, other centers in The Netherlands, and among members of the Dutch associations for parents of autistic individuals. Diagnostic information on the autistic individuals was obtained by means of an extensive phenotypic examination, including the Autism Diagnostic Interview Revised (ADI-R) [28, 31], Autism Diagnostic Observation Schedule (ADOS) [29, 30], The Vineland Adaptive Behavior Scales [46], and a medical assessment (screening for chromosomal abnormalities and fragile-X). Additional information was obtained from medical records. Reasons for exclusion were known medical disorders or sensory impairment.

For the present study, the first 30 families in which at least two children had a clinical diagnosis of ASD and met criteria on the ADI-R and ADOS were invited to take part and 28 families were willing to participate. Both parents and children older than 7 years of age and with an IQ of at least 70 were included. Additional psychometric data were obtained by administering a four-subtest short form of the Dutch version of the Wechsler Scales [24]. Among parents there is missing data on three fathers and two mothers who could not come to our center for assessment or where video recording of the assessment with the block design task failed. Twenty-five of the 56 children with a clinical diagnosis of ASD met the inclusion criteria (age $>7$ and IQ > 70). Two additional children were excluded, due to lack of cooperation during assessment or refusing video recording. Twelve of the participating individuals had a clinical diagnosis of autism and met criteria for autism on the ADI-R and the ADOS. Eleven individuals met criteria for a spectrum disorder on the ADI-R (defined as falling one point short on one of the domains) or ADOS. Their classifications on the basis of the DSM-IV were Autism $(n=1)$, Asperger Syndrome $(n=7)$ and PDD-NOS $(n=3)$.

As a comparison group for the individuals with ASD, typically developing children and adolescents were recruited from regular schools. They were screened by telephone for behavioral or psychiatric problems, or ASD in their family, and were then assessed with the Child Behavior Checklist (CBCL) and the Teacher Report Form (TRF) [1, 2, 50-52]. Individuals with psychiatric or behavioral problems or with first or second-degree family members with ASD were excluded $(n=3)$. Thirty-seven children participated in the study, but the data of one child was lost because the assessment was not visible on video. The clinical and the control group were matched for gender, mean age, and IQ.

As a comparison group for the parents of the children with ASD, parents of a child with down syndrome (DS) were recruited from the community through the Dutch Down syndrome Parent Association. The reason for choosing parents of children with another disability as a comparison group was because of research questions that were addressed in a separated study and not reported on in this paper. The parent groups were also matched for gender, mean age, and IQ. There were 30 control parent couples participating in the study, but missing data on three control parents: one father could not come to our center for assessment and videotaped data were lost for two mothers. Altogether, 167 individuals participated in our experiment.

All participants had normal or corrected to normal vision. The experimental protocol was approved by the Medical Ethics Committee of the University Medical Centre Utrecht and informed consent was obtained before testing from all parents and from children older than 12 years.

Table 1 shows the mean age of all individuals and Table 2 shows the mean IQ scores on the short form of the Wechsler scales for the subject groups. There were no significant differences in age, TIQ, VIQ or PIQ between any of the groups and their control groups. Also, no differences were found between the fathers and the mothers of the ASD and control groups.

\section{Materials}

Patterns from the task developed by Akshoomoff and Stiles [3] were used. Materials consist of the set of red

Table 1 Subject characteristics: mean age (and SD)

\begin{tabular}{|c|c|c|c|c|c|c|}
\hline & \multicolumn{3}{|c|}{ ASD group } & \multicolumn{3}{|c|}{ Control group } \\
\hline & \multirow[t]{2}{*}{$N$} & \multicolumn{2}{|l|}{ Age } & \multirow[t]{2}{*}{$N$} & \multicolumn{2}{|l|}{ Age } \\
\hline & & Mean & SD & & Mean & SD \\
\hline Children/adolescents & 23 & 17.1 & (7.9) & 36 & 16.8 & (7.9) \\
\hline Parents & 51 & 45.1 & (8.9) & 57 & 46.6 & (8.9) \\
\hline Fathers & 25 & 46.4 & (9.4) & 29 & 46.7 & (9.3) \\
\hline Mothers & 26 & 43.9 & (8.3) & 28 & 46.5 & (8.7) \\
\hline
\end{tabular}


Table 2 Subject characteristics: mean verbal (VIQ), performance (PIQ) and total intelligence (TIQ) (and SD) on the short form of the Wechsler scales

\begin{tabular}{|c|c|c|c|c|c|c|c|c|c|c|c|c|}
\hline & \multicolumn{6}{|c|}{ ASD group } & \multicolumn{6}{|c|}{ Control group } \\
\hline & \multicolumn{2}{|l|}{ VIQ } & \multicolumn{2}{|l|}{ PIQ } & \multicolumn{2}{|l|}{ TIQ } & \multicolumn{2}{|l|}{ VIQ } & \multicolumn{2}{|l|}{ PIQ } & \multicolumn{2}{|l|}{ TIQ } \\
\hline & Mean & SD & Mean & SD & Mean & SD & Mean & SD & Mean & SD & Mean & SD \\
\hline Children / adolescents (spectrum) & 103.8 & $(23.6)$ & 101.0 & $(19.2)$ & 102.6 & $(19.3)$ & 100.9 & $(16.5)$ & 98.7 & $(15.1)$ & 99.5 & (13.5) \\
\hline Parents & 105.3 & $(22.0)$ & 104.5 & $(17.2)$ & 105.7 & (19.9) & 103.3 & $(23.1)$ & 105.4 & (18.8) & 104.5 & (19.9) \\
\hline Fathers & 101.6 & $(22.9)$ & 102.5 & $(16.2)$ & 102.3 & $(20.2)$ & 106.6 & (23.9) & 105.6 & (17.6) & 106.7 & $(20.4)$ \\
\hline Mothers & 108.9 & $(20.8)$ & 106.5 & (18.3) & 108.9 & (19.5) & 99.8 & (22.2) & 105.1 & (20.4) & 102.2 & (19.5) \\
\hline
\end{tabular}

and white blocks of the Wechsler Scales. Each block has two red sides, two white sides and two sides that are half red and half white with the division oriented along the diagonal. The target-patterns were printed in red and white and collected in a binder. The first set of six patterns require four blocks $(2 \times 2$ squares $)$ to construct the pattern, the second set of 6 patterns require nine blocks $(3 \times 3$ squares $)$. There were two different pattern types: local and global patterns. For local patterns, perceptual cohesiveness (the number of block edges bordering on a neighboring block edged with the same color) was low, while in the global designs there was a maximum degree of perceptual cohesiveness. Within each set there were three local and three global patterns, which were presented in alternated order. Then a third and fourth set of presegmented patterns were presented. These patterns were exactly the same as in the first and second set and presented in the same order, but the block-edges were outlined with a black line. There were 24 patterns altogether plus a 4-blocks and a 9-blocks practice item.

\section{Procedure}

All participants were individually tested in a quiet room. First, the participants were shown four blocks and it was explained that all blocks were colored the same. The different sides of the blocks were shown and the practice item was presented. All participants were well able to construct the practice item. Then the task conditions were explained to the subject and they were encouraged to reconstruct the patterns as quickly as possible. The administration of the task was videotaped for later data analysis. Although the videos were used to measure construction time, a stopwatch was used during the task, to provide extra non-verbal pressure on the participants to reconstruct the patterns as quickly as they could. Participants were allowed maximally $90 \mathrm{~s}$ to construct the 4-block patterns and $180 \mathrm{~s}$ for the 9-block patterns. After six items the additional blocks were added, so that the subject had nine in total and a practice item with nine blocks was presented. Again, all participants were well able to construct this design.

\section{Scoring}

Before each test, a subject identification number was shown in front of the camera, which made it possible to code the videos blind to group status. Videotapes were coded by means of the "The Observer" [34]. This is a software system for recoding, coding and analyzing frequencies and durations of observed events. The use of this video tape analyses system enables coding behavior at different VCR playback speeds, while maintaining a proper time reference. This enables an exact coding (in hundredths of seconds) of the start and end of each occurrence of behavior.

For each design, the final result (whether the design was correctly reconstructed within the given time) and the time taken to reconstruct the pattern, was recorded. In addition, the number and type of errors made during reconstruction were recorded. Most of these errors were self-corrected within time limits. Three types of errors made during reconstruction were defined. Color errors refer to placing a block with the wrong colored side up (for instance red-side in stead of white-side). Matrix errors refer to breaking the $2 \times 2$ or $3 \times 3$ matrix by placing for instance four blocks on a row. Block rotation errors refer to red-white block placed with the diagonal oriented in the incorrect direction. The block design task was coded by two observers, who were blind to the diagnostic status of the participants. To test the inter-rater agreement of the coding, 33 videotapes $(=20 \%)$ were coded twice by the independent coders. The agreement corrected for chance was 0.72 (Cohen's Kappa) which is usually interpreted as substantial agreement [27].

\section{Data analysis}

The statistical package SPSS (version 11.5 for Windows) was used to analyze the data. The accuracy 
score was defined as the total number of patterns correctly reconstructed. The participants were well able to reconstruct the patterns correctly. Since the accuracy data were not normally distributed a 2-tailed Mann-Whitney $U$ was used to compare the mean number of correctly constructed designs between individuals from ASD families with individuals from the matched control group. The mean reconstruction time was defined as the mean time needed to reconstruct the patterns and was calculated for the different pattern types separately. Mean number of errors and error types made during reconstruction of the designs were calculated for the different pattern types separately, as well.

Additionally, $2 \times 2 \times 2 \times 2$ repeated measures analyses of variance (ANOVA) were performed with a between-factor Group (ASD vs. Control) to test for differences in reconstruction time, number of errors and type of errors between individuals from ASD families and control individuals. Within-subject factors all had two levels: Number (4-block vs. 9-block designs), Segmentation (unsegmented vs. segmented) and Pattern Type (local vs. global). Separate analyses were done for children and parent groups. In addition we compared the performance of the groups of father separately.

In case of a significant interaction, we tested partial interactions to determine the locus of the effect. In case an interaction with Group was found, partial interactions were tested to determine at which level of a specific factor, the groups differed. In addition, two planned comparisons were performed on reconstruction time between individuals from ASD families with individuals from the matched control group, using independent sample $t$ tests. First, we compared the groups on reconstruction time of the patterns in the most difficult condition requiring the highest level of mental segmentation ability (i.c. 9-blocks, global, unsegmented patterns). Second, a difference score was calculated, based on the methods used in the study of Happé, Briskman and Frith [19]. The difference score referred to the difference in reconstruction time between unsegmented en pre-segmented designs in the 9-block, global condition. All significance tests presented are two-tailed. Statistical significance was defined as $P<0.05$.

\section{Results}

The accuracy level, that is, the percentage of patterns constructed correctly, was high for all individuals. The mean number of correctly constructed designs was $23.8(\mathrm{SD}=0.4)$ in the ASD group and 23.4 $(\mathrm{SD}=0.8)$ in the control group. In parents the mean number of correctly constructed designs was 23.5
$(\mathrm{SD}=0.8)$ in the ASD-parent group and $23.3(\mathrm{SD}=$ $1.5)$ in the matched control group of parents. There was no significant difference between individuals with ASD and individuals from the control group in the number of correctly constructed designs. Also, no significant differences were found between the fathers or mothers of the individuals with ASD and the fathers or mothers of the individuals with DS.

\section{Reconstruction time in the children groups}

When comparing the group of individuals with ASD with the matched control group, analysis of variance revealed an interaction effect on the borderline of significance between Group $\times$ Number $\times$ Segmentation $\times$ Pattern Type $\mathrm{F}(1,57)=4.03, P=0.049$. Partial interactions showed a Group $\times$ Pattern Type effect in the unsegmented 9-block patterns $\mathrm{F}(1,57)=5.12$, $P=0.03$. However, when tested out, it was found that both groups reconstructed the local patterns in this condition significantly faster than the global patterns, although the difference was smaller in the ASD group (individuals with ASD: mean difference local-glo$\mathrm{bal}=12.2 \mathrm{~s}, t=4.66$; matched control group: mean difference local-global $=20.0 \mathrm{~s}, t=6,51$ ).

The planned comparisons revealed that there was no significant difference between the groups on the most difficult task condition (the 9-blocks, global, unsegmented patterns). The difference score (9blocks, global patterns in the unsegmented condition versus the segmented condition) was compared between groups. The difference in reconstruction time between these conditions did not reach significance when we compared the ASD group to the matched control group.

\section{Reconstruction time in parents}

For the groups of parents no significant interaction effect was found between any of the manipulations and the between-subjects factor Group (ASD parents vs. Control parents). In fact, the reconstruction times were very similar in both groups in all conditions as is shown in Fig. 1. For fathers, repeated measures also revealed no significant interaction effect between any of the manipulations and the between-subjects factor Group (ASD fathers vs. Control fathers). Planned comparisons also did not show differences.

\section{Errors made during construction}

An analysis of variance on total number of errors made during reconstruction revealed a significant interaction effect for Number $\times$ Group $F(1$, 


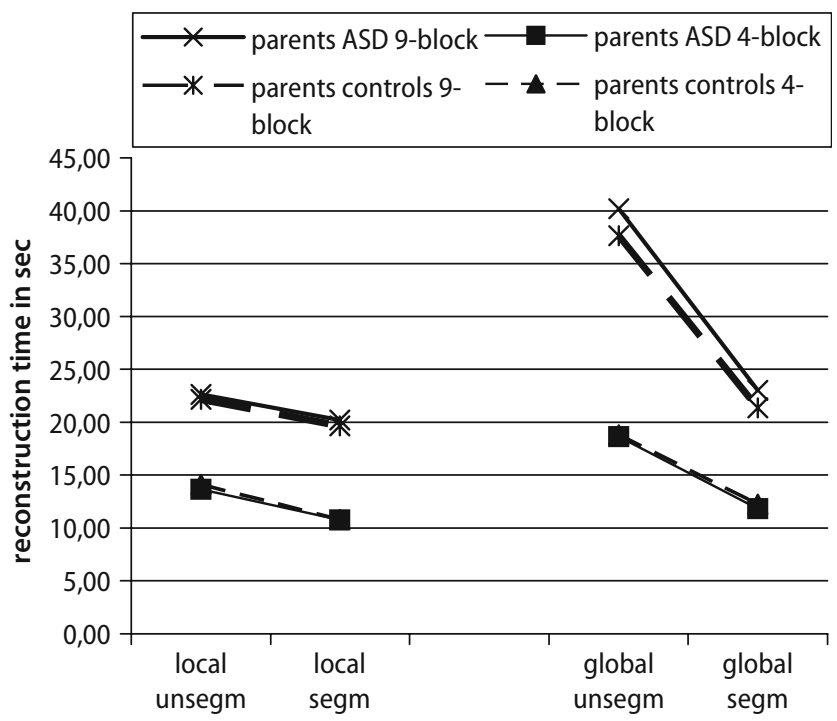

Fig. 1 Mean construction times for the local patterns (segmented vs. unsegmented condition) on the left and the global patterns (segmented vs. unsegmented condition) on the right, for parents of the ASD group and the parent control group

$57)=12.33, P<0.01$. Further analysis showed that there was a significant Group interaction effect for the 9-block designs only $\mathrm{F}(1,57)=13.1, P<0.01$, indicating that the individuals in the ASD group made significantly fewer errors (mean number of errors $=$ 1.86; $\mathrm{SD}=3.4)$ than the individuals in the control group (mean number of errors $=5.57 ; \mathrm{SD}=4.9$ ). In parents however, there were no group effects (mean number of errors 9-block condition parents ASD group $=4.1 ; \mathrm{SD}=4.5 ;$ parent control group $=3.6$; $\mathrm{SD}=5.5$ ).

Further analysis showed that there were specific kinds of errors made more often by individuals in the control group than by individuals with ASD. With respect to the number of Color errors we found no significant differences between the ASD-group (mean number of errors $=0.77 ; \mathrm{SD}=2.4$ ) and the matched control group (mean number of errors $=1.43$; $\mathrm{SD}=$ 2.1 ), nor between the parent groups. With respect to the number of Matrix errors, a significant interaction effect found was for Number $\times$ Group $F(1,57)=6.51$, $P<0.013$, but further analysis for the 4- and 9-block conditions showed no significant groups effect (mean number of errors 9-block condition ASD group = $0.45 ; \mathrm{SD}=1.1 ;$ control group $=1.11 ; \mathrm{SD}=1.8)$. No significant differences were found between the parent groups.

In addition, for the number of Block rotation errors made during reconstruction, a significant interaction effect was found for Number $\times$ Group $F(1$, $57)=8.52, P<0.01$. Further analysis showed that there was no interaction effect for the 4-block patterns between groups, but a significant group effect for the 9-block patterns $\mathrm{F}(1,57)=15.84, P<0.01$, indicating that the individuals with ASD made significantly fewer Block rotation errors (mean number of errors $=0.64 ; \mathrm{SD}=1.3)$ than the individuals in the control group (mean number of errors $=3.03$; $\mathrm{SD}=$ 3.3). In parents, there were no significant differences between groups (mean number of Block rotation errors 9-block condition parents ASD group $=1.9$; $\mathrm{SD}=2.4$; parent control group $=1.6 ; \mathrm{SD}=1.9$ ).

\section{Discussion}

This study investigated whether superior performance on a Block Design reconstruction task, could be identified in high-functioning individuals with ASD from multi-incidence families compared to a matched control group. In addition we investigated whether superior performance was found in the parents of the individuals with ASD compared to a matched control group of parents. Since our sample consisted of highfunctioning individuals with ASD and their parents, in whom these cognitive assets might be subtle, compared to matched control groups, we used a task that was thought to measure segmentation skills in the most sensitive way.

Contrary to our expectations, we found no significant differences in reconstruction time between highfunctioning individuals with ASD and matched control individuals. Absolute measures (planned comparison between reconstruction time on the most challenging condition of the task: 9-block unsegmented global patterns) did not reveal significant differences in performance between groups. We used two relative measures. Reconstruction time differences were compared between the local and global patterns in the unsegmented 9-block condition. Both groups however, were significantly faster in reconstructing the local than the global patterns, although the difference was somewhat smaller in the ASD group. In addition we did a planned comparison between reconstruction time of the 9-block global unsegmented and the segmented patterns. We did not find the difference in reconstruction time between these conditions to be significantly smaller in the ASD group than in the matched control group. Hence, there were no indications that the individuals with ASD were less aided by the pre-segmentation of the designs in comparison to the control group.

We expected that superior performance on the Block Design task would not only be reflected in the velocity in reconstructing a pattern but also in the accuracy with which the individuals position a block during the process of reconstruction. The assumption was that individuals with superior mental segmenta- 
tion abilities place a block in the correct position within a design right away. While by contrast, individuals with fewer mental segmentation skills, would more frequently place a block in an incorrect position initially and adjust that subsequently during the process of reconstruction. Indeed, we found that individuals with ASD made significantly fewer errors during the reconstruction of the 9-block patterns than control individuals. The differences between individuals with ASD and individuals from the matched control group were most striking in a specific type of error: block rotation errors. This error type refers to a diagonal block face rotated in an incorrect direction. In contrast, no differences were found between groups in color errors e.g. placing a red-sided block while a white-sided block was required or in matrix errors e.g. breaking the $2 \times 2$ or $3 \times 3$ matrix. This indicates that individuals with ASD do not perform better in choosing a block for a specific position per se. Their superiority however, is reflected in the condition that relies most on mental segmentation ability: the ability to decide exactly how and in which direction a double-colored block must be positioned within a design.

These findings are consistent with a study in which the Embedded Figures Test was used in a high-functioning sample of individuals with ASD and their parents. When looking at the number of errors during the process of searching for the correct shape, both individuals with ASD and their fathers pointed out significantly less incorrect shapes before finding the right one, compared to matched controls [11].

In the present study, we also looked at the performance of parents on the Block Design task, but did not find any evidence for superior performance in parents. Parents from ASD families were not faster than the control group of parents in reconstructing the designs, not even in the most strenuous pattern type condition. When looking at difference scores between local and global, or unsegmented and segmented patterns, in order to focus specifically on mental segmentation ability, parents of ASD families, did not stand out either. In addition no differences in the number or specific type of errors were found between the parent groups. Since it is previously found that behavioral characteristics of the broader phenotype are more common in males than in female relatives $[5,6,8,38,39,48]$ and since there is some evidence that this also extends to cognitive features [19], we looked at the performance of fathers separately. However, there were no differences between fathers from children with ASD and control fathers on any of the measures.

Taken together, we conclude that in our sample of high-functioning autistic individuals, we could not replicate weak central coherence as reflected in faster block design reconstruction performance in comparison to a matched control group. Neither absolute, nor relative measures revealed significant differences in reconstruction time between the two groups. This is in line with previous studies using different versions of Block Design tasks in high-functioning individuals with autism or Asperger's syndrome. In some of these studies a peak on the Block Design task compared to other tasks was relatively small [17, 20, 45], while in other studies it was not found at all [12, $35,36,41,49]$. Although there are also studies that reported significant higher scores on the Block Design task in high-functioning subjects compared to controls $[10,33]$ most studies found significant better performance in lower functioning individuals with autism but not in high-functioning individuals [32, $41,44]$. In the present study the number of times a block was placed in the correct location and orientation at first attempt appeared to be a more sensitive measure of weak central coherence or mental segmentation ability. Previous research showed that a larger number of errors made during the reconstructing block designs are associated with weaker visuospatial skills in typically developing children and adults $[25,26]$.

We found no evidence for a reflection of weak central coherence in superior mental segmentation skills in parents of autistic individuals, nor in fathers of autistic individuals separately. This is not in line with the study of Happé et al. [19], who found fathers of autistic individuals to be significantly faster on unsegmented patterns, but not on pre-segmented patterns, compared to fathers of typically developing children. Our results replicate however, the results of two studies not finding fathers nor mothers of autistic individuals to stand out on the Block Design task $[13,47]$.

The main limitation of our study is that our sample includes a relatively modest number of individuals, due to the fact that we recruited multi-incidence families. The lack of differences between groups might be a result of a lack of power. However, compared to studies that found superior block design performance in their sample, the size of our samples was not smaller than most of these studies. In addition, the reconstruction times of the parent groups are very much alike and do not suggest possible differences in performance that might become significant when adding a reasonable number of individuals to our sample.

A particular strength of this study lies in the measure used to assess block design reconstruction performance. We choose a task that made fine-tuned measuring of subtle differences in performance possible. Low perceptual cohesive (local) patterns and high perceptual cohesive (global) patterns, could be 
presented in alternated order without revealing prompts for a strategy to mentally segment the patterns. We did not only measure reconstruction time but the number and type of errors made during the process of reconstruction as well. Another strength of the present study is that we choose to use a software system designed to code observational data, in order to be able to score the data blind to group status. Therefore, all measurements were recorded on videotape. This method also made very accurate time measurements possible.

In conclusion, there are some indications for good mental segmentation abilities, as measured with a block design reconstruction task, shown by a lower number of errors made during reconstruction, in the high-functioning individuals with ASD compared with a matched control group. However, superior mental segmentation ability was not evident when looking at reconstruction time of the most difficult patterns or the extent to which subjects were aided by pre-segmentation. Furthermore, dexterous mental segmentation ability did not extend to the broader phenotype. Therefore, based on our data, we might conclude that it is not a neurocognitive marker or endophenotype useful in genetic studies.

\section{References}

1. Achenbach TM (1991a) Integrative guide for the $1991 \mathrm{cbcl} / 4-18$, ysr and trf profiles. University of Vermont Department of Psychiatry, Burlington

2. Achenbach TM (1991b) Manual for the child behavior checklist/4-18 and 1991 profile. University of Vermont Department of Psychiatry, Burlington

3. Akshoomoff NA, Stiles J (1996). The influence of pattern type on children's block design performance. J Int Neuropsychol Soc 2(5):392-402

4. American Psychiatric Association (2000) Diagnostic and statistical manual of mental-disorders, 4th edition text revised (DSM-IV). American Psychiatric Association, Washington, DC

5. Bailey A, Le Couteur A, Gottesman I, Bolton P, Simonoff E, Yuzda E, et al. (1995) Autism as a strongly genetic disorder: Evidence from a british twin study. Psychol Med 25(1):63-77

6. Bailey A, Palferman S, Heavey L, Le Couteur A (1998) Autism: the phenotype in relatives. J Autism Dev Disord 28(5):369-392

7. Baron-Cohen S, Hammer J (1997) Parents of children with asperger syndrome: What is the cognitive phenotype? J Cogn Neurosci 9(4):548554

8. Bolton P, Macdonald H, Pickles A, Rios P, Goode S, Crowson M, et al. (1994) A case-control family history study of autism. J Child Psychol Psychiatry 35(5):877-900

9. Caron MJ, Mottron L, Berthiaume C, Dawson M (2006) Cognitive mechanisms, specificity and neural underpinnings of visuospatial peaks in autism. Brain 129(Pt 7):1789-1802

10. Caron MJ, Mottron L, Rainville C, Chouinard S (2004) Do high functioning persons with autism present superior spatial abilities? Neuropsychologia 42(4):467-48
11. de Jonge MV, Kemner C, van Engeland H (2006) Superior disembedding performance of high-functioning individuals with autism spectrum disorders and their parents: The need for subtle measures. J Autism Dev Disord 36(5):677-683

12. Ehlers S, Nyden A, Gillberg C, Sandberg AD, Dahlgren SO, Hjelmquist E, et al. (1997) Asperger syndrome, autism and attention disorders: A comparative study of the cognitive profiles of 120 children. J Child Psychol Psychiatry 38(2):207-217

13. Fombonne E, Bolton P, Prior J, Jordan H, Rutter M (1997) A family study of autism: cognitive patterns and levels in parents and siblings. J Child Psychol Psychiatry 38(6):667-683

14. Freeman BJ, Ritvo ER, Needleman R, Yokota A (1985) The stability of cognitive and linguistic parameters in autism: A five-year prospective study. J Am Acad Child Psychiatry 24(4):459464

15. Frith U (1989) Explaining the enigma Basil. Blackwell, Oxford

16. Frith U, Happe F (1994) Autism: beyond "theory of mind". Cognition 50:115-132

17. Gilchrist A, Green J, Cox A, Burton D, Rutter M, Le Couteur A (2001) Development and current functioning in adolescents with asperger syndrome: A comparative study. J Child Psychol Psychiatry 42(2):227-240

18. Goldstein G, Beers SR, Siegel DJ, Minshew NJ (2001) A comparison of wais-r profiles in adults with high-functioning autism or differing subtypes of learning disability. Appl Neuropsychol 8(3):148-154
19. Happe F, Briskman J, Frith U (2001) Exploring the cognitive phenotype of autism: Weak "central coherence" in parents and siblings of children with autism: I. Experimental tests. J Child Psychol Psychiatry 42(3):299-307

20. Happe FG (1994) Wechsler iq profile and theory of mind in autism: A research note. J Child Psychol Psychiatry 35(8):1461-1471

21. Happé F, Frith U (2006) The weak coherence account: detail-focused cognitive style in autism spectrum disorders. J Autism Dev Disord 36(1):5-25

22. Hill EL, Frith U (2003) Understanding autism: Insights from mind and brain. Philos Trans R Soc Lond B Biol Sci 358(1430):281-289

23. Kaland N, Mortensen EL, Smith L (2007) Disembedding performance in children and adolescents with asperger syndrome or high-functioning autism. Autism 11(1):81-92

24. Kort W, Schittekatte M, Dekker PH, Verhaeghe P, Compaan EL, Bosmans $\mathrm{M}$, et al. (2005) Wechsler intelligence scale for children-third edition, dutch version. Harcourt, The Netherlands

25. Kramer JH, Kaplan E, Blusewicz MJ, Preston KA (1991) Visual hierarchical analysis of block design configural errors. J Clin Exp Neuropsychol 13(4):455-465

26. Kramer JH, Kaplan E, Share L, Huckeba W (1999) Configural errors on wisc-iii block design. J Int Neuropsychol Soc 5(6):518-524

27. Landis JR, Koch GG (1977) The measurement of observer agreement for categorical data. Biometrics 33:159-174

28. Lord C, Pickles A, McLennan J, Rutter M, Bregman J, Folstein S, et al. (1997) Diagnosing autism: Analyses of data from the autism diagnostic interview. J Autism Dev Disord 27(5):501-517 
29. Lord C, Risi S, Lambrecht L, Cook EH Jr, Leventhal BL, DiLavore PC, et al. (2000) The autism diagnostic observation schedule-generic: a standard measure of social and communication deficits associated with the spectrum of autism. J Autism Dev Disord 30(3):205-223

30. Lord C, Rutter M, Goode S, Heemsbergen J, Jordan $\mathrm{H}$, Mawhood L, et al. (1989) Autism diagnostic observation schedule: a standardized observation of communicative and social behavior. J Autism Dev Disord 19(2):185-212

31. Lord C, Rutter M, Le Couteur A (1994). Autism diagnostic interview-revised: a revised version of a diagnostic interview for caregivers of individuals with possible pervasive developmental disorders. J Autism Dev Disord 24(5):659685

32. Mayes SD, Calhoun SL (2003) Analysis of wisc-iii, stanford-binet:Iv, and academic achievement test scores in children with autism. J Autism Dev Disord 33(3):329-341

33. Mottron L (2004) Matching strategies in cognitive research with individuals with high-functioning autism: Current practices, instrument biases, and recommendations. J Autism Dev Disord 34(1):19-27

34. Noldus LPJJ (1991) The observer: A software system for collection and analysis of observational data. Behav Res Methods Instrum Comput 23:415429

35. Ozonoff S, Pennington BF, Rogers SJ (1991a) Executive function deficits in high-functioning autistic individuals: Relationship to theory of mind. J Child Psychol Psychiatry 32(7):1081-1105
36. Ozonoff S, Rogers SJ, Pennington BF (1991b) Asperger's syndrome: evidence of an empirical distinction from highfunctioning autism. J Child Psychol Psychiatry 32(7):1107-1122

37. Pellicano E, Maybery M, Durkin K, Maley A (2006) Multiple cognitive capabilities/deficits in children with an autism spectrum disorder: "weak" central coherence and its relationship to theory of mind and executive control. Dev Psychopathol 18(1):77-98

38. Pickles A, Bolton $\mathrm{P}$, Macdonald $\mathrm{H}$, Bailey A, Le Couteur A, Sim CH, et al. (1995) Latent-class analysis of recurrence risks for complex phenotypes with selection and measurement error: a twin and family history study of autism. Am J Hum Genet 57(3):717-726

39. Pickles A, Starr E, Kazak S, Bolton P, Papanikolaou K, Bailey A, et al. (2000) Variable expression of the autism broader phenotype: findings from extended pedigrees. J Child Psychol Psychiatry 41(4):491-502

40. Piven J, Palmer P (1997) Cognitive deficits in parents from multiple-incidence autism families. J Child Psychol Psychiatry 38(8):1011-1021

41. Ropar D, Mitchell P (2001) Susceptibility to illusions and performance on visuospatial tasks in individuals with autism. J Child Psychol Psychiatry 42(4):539-549

42. Rumsey JM, Hamburger SD (1988) Neuropsychological findings in highfunctioning men with infantile autism, residual state. J Clin Exp Neuropsychol 10(2):201-221

43. Rutter M (2005) Aetiology of autism: findings and questions. J Intellect Disabil Res 49(Pt 4):231-238

44. Shah A, Frith U (1993) Why do autistic individuals show superior performance on the block design task? J Child Psychol Psychiatry 34(8):1351-1364
45. Siegel DJ, Minshew NJ, Goldstein G (1996) Wechsler iq profiles in diagnosis of high-functioning autism. J Autism Dev Disord 26(4):389-406

46. Sparrow SS, Balla DA, Cicchetti DV (1984) Vineland adaptive behavior scales. Interview edition. Survey form manual. American Guidance, Washington, DC

47. Szatmari P, Jones MB, Tuff L, Bartolucci G, Fisman S, Mahoney W (1993) Lack of cognitive impairment in firstdegree relatives of children with pervasive developmental disorders. J Am Acad Child Adolesc Psychiatry 32(6):1264-1273

48. Szatmari P, MacLean JE, Jones MB, Bryson SE, Zwaigenbaum L, Bartolucci $\mathrm{G}$, et al. (2000) The familial aggregation of the lesser variant in biological and nonbiological relatives of pdd probands: a family history study. J Child Psychol Psychiatry 41(5):579-586

49. Szatmari P, Tuff L, Finlayson MA, Bartolucci G (1990) Asperger's syndrome and autism: neurocognitive aspects. J Am Acad Child Adolesc Psychiatry 29(1):130-136

50. Verhulst FC, Van der Ende J, Koot HM (1996) Manual for the child behavior checklist (in dutch). Department of Child and Adolescent Psychiatry, Erasmus Medical Centre/Sophia, Rotterdam

51. Verhulst FC, Van der Ende J, Koot HM (1997) Manual for the teacher's report form (in dutch). Department of Child and Adolescent Psychiatry, Erasmus Medical Centre/Sophia, Rotterdam

52. Verhulst FC, Van der Ende J, Koot HM (1997) Manual for the youth self-report (in dutch). Department of Child and Adolescent Psychiatry, Erasmus Medical Centre/Sophia, Rotterdam 